Utah State University

DigitalCommons@USU

$12-2020$

\title{
Effects of Effort Training on Effort-Based Impulsive Choice
}

Sara Peck

Utah State University

Follow this and additional works at: https://digitalcommons.usu.edu/etd

Part of the Psychology Commons

\section{Recommended Citation}

Peck, Sara, "Effects of Effort Training on Effort-Based Impulsive Choice" (2020). All Graduate Theses and Dissertations. 7997.

https://digitalcommons.usu.edu/etd/7997

This Thesis is brought to you for free and open access by the Graduate Studies at DigitalCommons@USU. It has been accepted for inclusion in All Graduate Theses and Dissertations by an authorized administrator of DigitalCommons@USU. For more information, please contact digitalcommons@usu.edu.

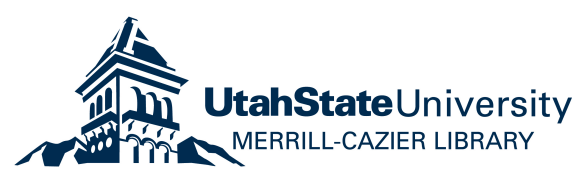


EFFECTS OF EFFORT TRAINING ON EFFORT-BASED IMPULSIVE CHOICE

by

Sara Peck

A thesis submitted in partial fulfillment of the

requirements for the degree

of

MASTER OF SCIENCE

in

Psychology

Approved:

Gregory Madden, Ph.D.

Major Professor

Timothy Shahan, Ph.D.

Committee Member

Katherine Brown, Ph.D.

D. Richard Cutler, Ph.D.

Committee Member Interim Vice Provost

of Graduate Studies

UTAH STATE UNIVERSITY

Logan, Utah 
Copyright (C) Sara Peck 2020

All Rights Reserved 


\begin{abstract}
Effects of Effort Training on Effort-Based Impulsive Choice
\end{abstract}

by

Sara Peck, Master of Science

Utah State University, 2020

Major Professor: Dr. Gregory Madden

Department: Psychology

Because impulsive decision-making is correlated with many maladaptive tendencies, researchers have increasingly studied methods for reducing impulsive choice. These efforts have primarily focused on increasing choices of larger, more-delayed rewards. A second type of impulsive choice is selecting a smaller, less-effortful reward over a larger, more-effortful one. Little nonhuman research has examined experimental methods for reducing effort-based impulsive choice. Within the realm of delay-based impulsive choice, extended exposure to reinforcer delays has proven effective in reducing impulsive choices in rats. The current study took a similar tac by evaluating if reductions in effortbased impulsive choice could be achieved by providing rats with extended exposure to high-effort training, i.e., reinforcement contingencies requiring a large number of responses. Male rats were randomly assigned to one of two groups: (1) high-effort training (fixed-ratio 50) or (2) low-effort training (fixed-ratio 1). Following training, both groups completed three tests of effort-based impulsive choice. There was an initial effect of HE training on effort-based impulsive choice, however the training effect quickly diminished across sessions for two out of the three tests. Possible explanations for the lack of lasting effect are discussed. 


\section{PUBLIC ABSTRACT \\ Effects of Effort Training on Effort-Based Impulsive Choice}

Sara Peck

Because impulsive decision-making is correlated with many maladaptive tendencies, researchers have increasingly studied methods for reducing impulsive choice. Most research in this area has focused on reducing delay-based impulsive choice, as measured by choices between smaller, immediate or larger, delayed rewards. A second type of impulsive choice is selecting a smaller, less-effortful reward over a larger, more-effortful one. Little nonhuman research has examined experimental methods for reducing effortbased impulsive choice. Within the realm of delay-based impulsive choice, extended exposure to reinforcer delays has proven effective in reducing impulsive choices in rats. The current study took a similar tac by evaluating if reductions in effort-based impulsive choice could be achieved by providing rats with extended exposure to high-effort training, i.e., reinforcement contingencies requiring a large number of responses. Male rats were randomly assigned to one of two groups: (1) high-effort training (fixed-ratio 50) or (2) low-effort training (fixed-ratio 1). Following training, both groups completed three tests of effort-based impulsive choice. There was an initial effect of HE training on effort-based impulsive choice, however the training effect quickly diminished across sessions for two out of the three tests. Possible explanations for the lack of lasting effect are discussed. 


\section{CONTENTS}

Page

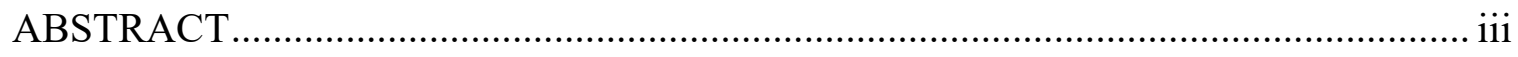

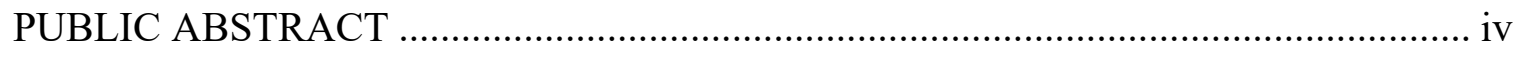

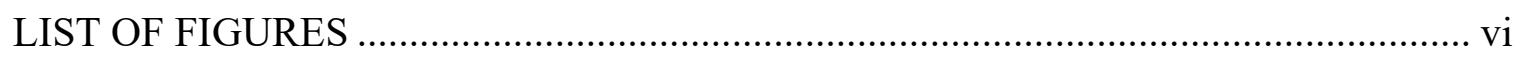

CHAPTER

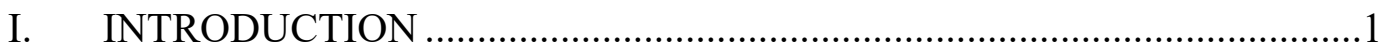

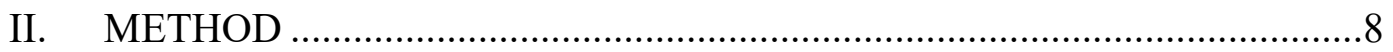

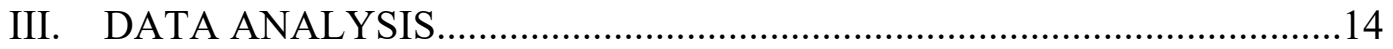

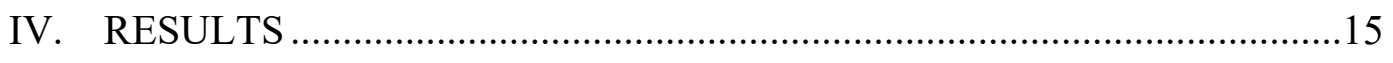

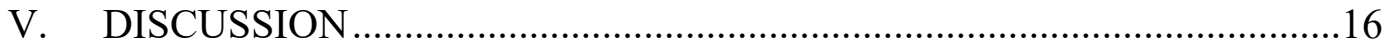

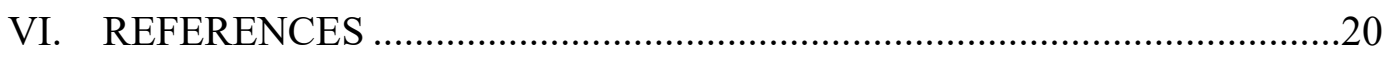

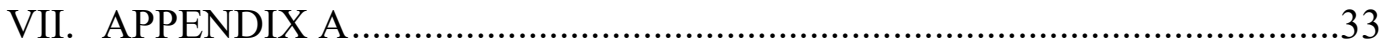




\section{LIST OF FIGURES}

Figure $\quad$ Page

1. Effort-Based Impulsive Choice Task Visual .............................................30

2. Effort-Based Impulsive Choice Tasks I \& III .................................................31

3. Effort-Based Impulsive Choice Tasks Stable Sessions .................................32 


\section{CHAPTER I}

\section{INTRODUCTION}

Impulsive decision-making may be defined as a maladaptive preference for a smaller, low-cost reward over a larger, higher-cost reward (Floresco, Tse, \& GhodsSharifi, 2008; Odum, 2011a; Sugiwaka \& Okouchi, 2004). Studies of impulsive decision making and discounting of costly rewards usually operationalize costs as delays (Odum, 2011b) but some studies have specified costs as effort expenditures (e.g., Mitchell, 2004). Steeply discounting the value of delayed rewards is correlated with behaviors negatively impacting human health; e.g., substance use (Mackillop et al., 2011), pathological gambling (Alessi \& Petry, 2003), obesity (Amlung, Petker, Jackson, \& Balodis, 2016) and suicide (Dombrovski et al., 2012). Less research has evaluated the behavioral correlates of steeply discounting the value of effortful rewards, perhaps because a standardized effort-discounting task has not been developed for humans.

Outside of the discounting domain, a tendency toward low effort-expenditures is predictive of poor academic performance (Duckworth \& Gross, 2014; Duckworth, Peterson, Matthews, \& Kelly, 2007; Weiner, 1985), giving up when faced with a challenge (Dweck, 2010), aversion to hard work (Eisenberger, 1992; Weiner, 1985), an inclination to smoke cigarettes and abuse drugs or alcohol (Quinn, Brandon, \& Copeland, 1996), and cigarette smoking relapse (Brandon et al., 2003). By contrast, high-effort tendencies are related to honesty (Eisenberger \& Masterson, 1983) and tolerance for aversive events experienced while pursuing superior outcomes (Eisenberger, Weier, Masterson, \& Theis, 1989). Effort expenditure has been operationalized in a variety of ways, including cognitive effort (e.g., anagram task; Eisenberger, Heerdt, Hamdi, Zimet, 
\& Bruckmeir, 1979b), physical effort (e.g., force exerted; Boyagian \& Nation, 1981) and task persistence when faced with operant extinction (Eisenberger, Carlson, Guile, \& Shapiro, 1979a; Wenrich, Eckman, Moore, \& Houston, 1967; see Eisenberger, 1992 for review).

Because of the maladaptive behaviors associated with both delay- and effortbased impulsive decision-making, researchers have begun to explore methods for reducing impulsive choice. Most of these efforts have focused on reducing delay discounting and delay-based impulsive choice (for reviews see Gray \& MacKillop, 2015; Koffarnus, Jarmolowicz, Mueller, \& Bickel, 2013; Rung \& Madden, 2018; Smith, Panfil, Bailey, \& Kirkpatrick, 2019). A wide variety of context manipulations have been shown to reduce delay-based impulsive choice in human and nonhuman subjects (e.g., Kowal \& Faulkner, 2016; Mazur, 2012; van der Wal et al., 2013; Wu \& He, 2012) but lasting effects observed in baseline testing conditions (i.e., when context is no longer manipulated) have only been explored in nonhumans (e.g., Logue \& Mazur, 1981; Smith, Marshall \& Kirkpatrick, 2015). In the Madden lab, long-lasting reductions in rats' delaybased impulsive choices have been reliably observed following extended experience with delayed-reinforcement contingencies (Renda \& Madden, 2016; Rung, Buhusi, \& Madden, 2018; Renda, Rung, Hinnenkamp, Lenzini, \& Madden, 2018; Peck et al., 2019; Stein et al., 2013, Stein, Renda, Hinnenkamp, \& Madden, 2015). Such findings encourage the exploration of methods for producing comparable long-lasting reductions in effort-based impulsivity. These reductions in impulsive choice are potentially important because, as noted above, maladaptive behaviors are correlated with steep delay discounting and the tendency toward low effort-expenditure. If reductions in these 
impulsivities are preventative of maladaptive behaviors such as substance-used disorders, then identifying effective and efficient methods for reducing impulsive decision-making could significantly impact public health.

If exposure to reinforcement delays reduces delay-based impulsive choice, then the most obvious candidate for reducing effort-based impulsivity is exposure to higheffort reinforcement contingencies. High-effort training was a staple in the learned industriousness literature (see Eisenberger, 1992 for review), although most of this work focused on the effects of high-effort training on task persistence in single-operant tasks (e.g. Eisenberger, Terborg, \& Carlson, 1979; Eisenberger et al., 1997a, 1997b). For example, Eisenberger, Carlson, Guile, and Shapiro (1979) reported that rats given a history of reinforcement for running five times up and down a runway shuttle (high-effort group) subsequently exerted more effort in a lever-pressing task conducted in a different chamber, when compared with a low-effort group required to run one-fifth this distance or a control group given no runway exposure prior to testing. In addition, only the higheffort group increased their rate of lever pressing when compared with their own prerunway baseline rates. These effects were replicated when effort training was completed on the lever (increasing force requirements) and testing was conducted in the runway.

Speaking to the external validity of this nonhuman research, Eisenberger frequently reported similar outcomes in single-operant studies enrolling human participants (e.g., Eisenberger \& Adornetto, 1986; Eisenberger et al., 1979b; Eisenberger, Leonard, Carlson, \& Park, 1979c; Eisenberger \& Leonard, 1980; Eisenberger, Masterson, \& McDermitt, 1982; Eisenberger \& Masterson, 1983; Eisenberger, Mitchell, \& Masterson, 1985; Eisenberger, Terborg, \& Carlson, 1979). For example, when 
Eisenberger et al. (1979b) gave psychiatric patients with depression a history of reinforcement for completing 4-5 cleaning/maintenance tasks, they subsequently worked harder on a card-sorting task than either a group given reinforcers after each cleaning/maintenance task, or a control group which was treated as normal in the ward. Similar beneficial outcomes of learned industriousness training in human participants have been reported by researchers outside of the Eisenberger lab (e.g. Boyagian \& Nation, 1981; Flora \& Pavlik, 1990; Nation, Cooney, \& Gartrell, 1979; Pittenger \& Pavlik, 1988).

While most of the learned industriousness work was conducted in single-operant test conditions, some studies have reported reductions in effort-based impulsive choice following high-effort training (e.g. in humans: Eisenberger \& Adornetto, 1986;

Eisenberger et al., 1985; and in nonhumans: Eisenberger et al., 1989). In one of the better controlled of these human studies, Eisenberger et al. (1985) asked 7-8 year-old children to choose between high pay (3 cents) for completing an effortful task (copying nonsense words) or less pay ( 2 cents) for simply waiting (a no-effort "task" with time to payment equalized to the effortful alternative). Children given a history of reinforcement for exerting high effort in tasks such as counting objects, matching shapes, and memorizing stimuli more often chose the high-pay effortful alternative, when compared with children with a low-effort history or no prior training (control group).

Far less nonhuman research has examined the effects of effort training on effortbased impulsive choice. Indeed, the only study I am aware of that has examined this is Eisenberger et al. (1989). In this study, rats were randomly assigned to receive food following every runway shuttle (low-effort group), every fifth shuttle (high-effort group), 
or to receive non-contingent food at intervals yoked to the high-effort group (control group). Following 18 training/yoked sessions, rats completed 12 effort-based impulsive choice test-sessions in which they chose between a low-effort alternative (FR 7 on a lowforce lever for 1 pellet) and a high-effort alternative (FR 7 on a high-force lever for 2 pellets). High-effort trained rats preferred the larger, more-effortful reward (61.1\% choice $^{1}$ ) significantly more than during their pre-training assessment (41.9\%) and more than the low-effort (43.2\%) or control (44.2\%) groups' post-training preferences.

The research thus far on learned industriousness and effort-based impulsive choice, while groundbreaking, is limited in three ways. First, in human and nonhuman studies, post-training assessments of preference for larger more-effortful rewards were evaluated at a single effort-requirement, so questions about the upper- and lower-range of effort requirements at which the learned-industriousness effect will be observed has not been evaluated. Are high-effort trained rats, for example, more likely to choose the larger, more-effortful reward only at the effort requirement arranged in training, or does this effect generalize to more demanding requirements? Modern effort-based impulsive choice assessments evaluate choice at a parametric range of effort requirements (e.g. Floresco et al., 2008). In the current study, rats' choice for low versus high effort was assessed at $1,15,30$, and 60 presses for the larger reward alternative.

Second, the Eisenberger studies provided brief durations of effort-training and evaluated only short-term effects of this training on effort-based impulsive choice. For example, in his human studies, high-effort training typically did not exceed three sessions, and testing was conducted in a single session (e.g. Eisenberger et al., 1985).

\footnotetext{
${ }^{1}$ Average of the final 6 sessions, here and below.
} 
The duration of effort-training in Eisenberger's nonhuman choice experiment (Eisenberger et al., 1989) was similarly brief. Although 18 sessions of training were arranged, much of that training was spent gradually increasing the effort requirement; only four training sessions were conducted at the terminal response requirement (5 runway shuttles). In addition to a brief training-duration, their assessment of effort-based impulsive choice was similarly limited. Impulsive choice was evaluated over a total of only 96 trials ( 8 choices per session). Given this brevity, it is noteworthy that a decreasing trend is observed over the final sessions in the high-effort group's preference for the larger, more-effortful reward. No such trend is observed in any other group. Whether the training effect would have proven robust over further testing is unknown. In the current research, I conducted an effort-based impulsive choice assessment that lasted a minimum of fifteen sessions (360 trials).

A third potential limitation is specific to the one nonhuman study in which the effects of learned industriousness have been examined in an effort-based impulsive choice procedure (Eisenberger et al., 1989). In that study, rats chose between completing an FR 7 on a low-force lever (1 pellet) and an FR 7 on a high-force lever (2 pellets). If rats could obtain food more quickly by choosing the low-force alternative, then effort and delay were confounded in the choice phase; i.e., the larger, more-effortful reward was also the larger-later reward. Modern effort-based impulsive choice procedures disentangle these costs by adding an equalized delay to food delivery when the smaller, low-effort alternative is selected (e.g., Floresco et al., 2008). The delay to reinforcement on the low-reward lever is yoked to the average time it takes to complete the FR 
requirement on the high-reward lever. In the current research, this yoking procedure was used for assessing effort-based impulsive choice.

To summarize, prior research has demonstrated that a brief course of high-effort training can increase humans' and rats' preferences for larger, more-effortful rewards (e.g. Eisenberger et al. 1989; Eisenberger et al., 1985). However, these demonstrations of reduced effort-based impulsive choice have evaluated only short-term effects. There are several reasons to conduct further research with nonhuman subjects. First, the benefits of learned industriousness training in nonhumans have been replicated in humans (Eisenberger et al., 1985; Eisenberger et al., 1979b; see Eisenberger, 1992 for review). Therefore, the current study may help to develop interventions that aim to reduce impulsive choice in humans. Second, extended delay-exposure training has produced replicable and lasting effects in rodent studies in the Madden lab (Peck et al., 2019; Renda \& Madden, 2016; Renda et al., 2018; Rung, Buhusi, \& Madden, 2018; Stein et al., 2013, Stein et al., 2015). Therefore, similar long-lasting effects might be expected if rats are given an extended course of high-effort training. Third, a tendency to exert low-effort in single-operant tasks is correlated with substance use (Quinn, Brandon, \& Copeland, 1996) and is predictive of cigarette smoking relapse (Brandon et al., 2003; Brown et al., 2002; Brown et al., 2004; Brown et al., 2009). If high-effort training proves effective in reducing effort-based impulsive choice in rats, future studies could examine if this training (a) reduces rats' drug self-administrations when choosing between a low-effort drug reward and a high-effort non-drug reward or (b) increases engagement with exercise as an alternative to drug-taking (Cosgrove, Hunter, Carroll, 2002; Larson \& Carroll 2005; Zlebnik \& Carroll, 2015a; Zlebnik \& Carroll, 2015b). Finally, and as outlined above, 
standard, well-controlled, and comparable procedures for measuring effort-based impulsive choice exist in the nonhuman literature (i.e. Floresco et al., 2008). Therefore, effort-based impulsive choice was evaluated without confounded delay on the smaller, less-effortful alternative, and at a range of effort requirements.

This study aimed to determine the effect of high-effort training on effort-based impulsive choice, with proper delay controls and at a range of effort requirements. Adolescent rats were matched by weight, then randomly assigned to high-effort or loweffort training groups. Rats in the high-effort group underwent extensive (i.e. 120 sessions) training at high effort requirements (i.e. FR 50). Low-effort rats completed the same number of training sessions but received immediate reinforcement upon a single lever press; inter-food intervals were yoked to the high-effort group. After these training sessions, effort-based impulsive choice was assessed using a variation of the Floresco et al. (2008) procedure. Effort was operationalized as an FR requirement instead of a force requirement because (1) increasing the latter can cause rats to alter their topography of lever pressing (e.g. using the weight of upper body to press the lever, instead of pressing it with a paw) and (2) increasing a lever's force requirement increases the number of unmeasured sub-criterion presses (Pinkston \& Libman, 2017). Both of these issues decrease the correlation between the arranged effort requirement and effort actually exerted. 


\section{CHAPTER II}

\section{Method}

\section{Subjects}

Thirty experimentally naïve male Wistar rats, approximately 35 days old at the start of the experiment, served as subjects. Male rats were selected, instead of female rats, because the delay-exposure effect has been shown only in male rats thus far. Sample size was determined by a power analysis ( $\mathrm{G}^{*}$ Power Statistics) based on the average of effect sizes reported in delay-exposure training experiments (i.e., Renda \& Madden, 2016; Renda et al., 2018; Stein et al., 2013, Stein et al., 2015). Specifically, the effect of delay exposure training compared to immediacy exposure training was used, as those groups most closely mirrored the comparison groups in this study. A delay-exposure effect size was used, instead of that reported by Eisenberger et al. (1989) because the data needed to calculate their effect size were not reported. G*Power recommended 10 rats per group, which was increased by $50 \%$ because of unknowns about the likely effect of the effort training arranged.

Rats were kept at $85 \%$ of their growth-curve projected free-feeding weight and had free access to water in their home-cages. Rats were single-housed and provided with cage enrichment in the form of nesting paper. Rats were matched by weight and then block-randomly assigned to either the high-effort or low-effort training groups. One rat was removed from the study due to failure to ever complete an FR50 in high-effort training. Two rats passed away unexpectedly during the effort training phase, due to reasons unrelated to the study.

\section{Apparatus}


Sessions were conducted in eight identical, sound-attenuating operant chambers (MED Associates; St. Albans, VT). Chambers were equipped with a white-noise speaker, house-light, and three retractable levers (two on the front-wall and one on the rear), all of which were located below white 28-V DC cue lights. Bioserv multigrain pellets were delivered by a pellet dispenser. Stimulus events and behavior was controlled and recorded using MED Associates software.

\section{Procedures}

Acquisition. Rats acquired the lever-press response through a modified autoshaping procedure arranged on the rear-wall lever. Sessions consisted of 20 trials, each ending with the delivery of two 45-mg multigrain food pellets. Trials began with the insertion of the rear-wall lever and the illumination of its cue light. A single press of the lever produces the food reinforcer. If a press did not occur within $10 \mathrm{~s}$, the pellets were delivered non-contingently. The first five trials were initiated once per $90 \mathrm{~s}$. The remaining 15 trials began in the same way, but a single lever press was required to produce food and initiate the inter-trial interval. Sessions ended once 20 trials were completed or after 2 hours elapsed, whichever came first. Acquisition sessions continued until at least $90 \%$ of the available food deliveries were obtained by lever-pressing in two consecutive sessions.

Effort Training Phase. After rats met the lever acquisition criterion, they completed 120 sessions of their respective training protocols (described below). Across groups, training sessions were composed of 80 trials, each initiated with the insertion of the rear-wall lever and illumination of its cue light. Completing the response requirement retracted the lever and resulted in the immediate delivery of 2 food pellets in the 
receptacle on the front wall. Failure to press a lever within $20 \mathrm{~s}$ initiated a blackout for the remainder of the trial, which was scored as an omission; omitted trials were repeated. Sessions terminated after 80 trials or $120 \mathrm{~min}$, whichever occurred first.

High-effort (HE) training group ( $\boldsymbol{n}=14)$. For rats assigned to the HE group, the response requirement imposed on the rear-wall lever was gradually increased, according to the following progression of FR values: 2, 5, 10, 20, 30, 40, and 50. Rats completed 5 trials per FR increase, before reaching the terminal FR value. This within-session sequence of escalating FR values continued until two consecutive sessions were completed in which the rat earned at least 74 of the 80 available reinforcers. Trials initiated every $60 \mathrm{~s}$, unless the response requirement had not yet been completed. In which case, the trial extended until the reinforcement contingency was met or the session ended (after 121 mins), whichever came first. If the response requirement was completed before the nominal 60-s trial duration ended, the post-food interval was spent in blackout.

Low effort (LE) training group (n=13). LE rats completed an FR1 on the rearwall lever and received immediate food. After food was delivered, a blackout period was imposed until the start of the next trial. The blackout duration was programed and adjusted daily so that inter-trial intervals were yoked to the median obtained interreinforcer intervals in the HE group. At session 45 of training, the HE group's median inter-reinforcer intervals stabilized at 23-s and this value was used for the remainder of LE training.

Amount Discrimination Testing. After HE or LE training, rats completed 60trial sessions in which they chose between 1 and 3 pellets. Lever assignment was counterbalanced within groups. Trials began with the insertion of the rear-wall lever and 
illumination of its cue light. When that lever was pressed once, the two side-levers were inserted on the front-wall, with illumination of corresponding cue lights above each lever. For HE rats, pellets were obtained by completing an FR50 on both the low-reward and high-reward levers. LE rats had to complete an FR1 for either alternative. Each session was composed of four blocks of 12 trials each. Blocks began with six forced-choice trials (three on each lever), in which only one side-lever was inserted after the rear-wall lever was pressed. The remaining six trials were free-choice trials in which both side-levers were inserted. Forced-choice trials ensured that rats had experience with the contingencies operative on each lever within the block before making free choices. Failure to press a lever once within $20 \mathrm{~s}$ of any trial initiated a blackout for the remainder of the trial, which was scored as an omission. Trials, whether completed or not, were separated by a 20 -s blackout during which all lights were off and all levers retracted. Trial blocks were separated by comparable blackout periods lasting 5 mins. Rats passed the amount discrimination phase once they completed two consecutive sessions with at least $90 \%$ choice allocated to the high-reward lever.

Effort-Based Impulsive Choice Test I. To evaluate the effects of HE-training on effort-based impulsive choice, a variation of the effort-discounting with equivalent delays task (Floresco et al., 2008) was used (see Figure 1). This task was identical to the amount discrimination task, except the FR requirement on the high-reward lever increased across trial blocks: FR 1, 15, 30, and 60. Thus, in the first trial block, rats chose between completing an FR1 for 1 pellet (low-reward lever) and an FR 1 for 3 pellets (high-reward lever). Choice in the first trial block allowed continued assessment of the rats' sensitivity to the difference in reinforcer magnitude, with effort requirements being 
equal. Rats were returned to amount discrimination testing if, during this phase, preference for the high-reward alternative fell below $80 \%$ in the first trial block for two consecutive sessions. In the second trial block, the choice was between an FR1 for 1 pellet and FR15 for 3 pellets, and so on.

Throughout the session, the low-reward lever always operated according to an FR1. However, to ensure that choices were made based on differences in the FR values, and not differences in time to food, the 1-pellet reinforcer for low-reward lever presses was delivered after a signaled delay yoked to the average time it took the individual rat to complete the FR arranged on the high-reward lever during forced-choice trials of the trial block (Floresco et al., 2008; Ghod-Sharifi \& Floresco, 2010; Ghods-Sharifi, Onge, \& Floresco, 2009; Shafiei, Gray, Viau, \& Floresco, 2012). See Appendix for a list of the yoked delays in trial blocks 2-4, across the final five sessions in this phase. For example, if an average of $18 \mathrm{~s}$ elapsed between lever insertion and completion of the FR 30 in the forced-choice high-reward trials, the time to food following a low-reward lever press was $18 \mathrm{~s}$; the cue light above the chosen lever remained on during this interval (see Figure 1). To accomplish this yoking, the first three forced-choice trials were always completed on the high-effort alternative.

Rats completed a minimum of 15 sessions in this phase, and continued until preference stabilized. Choice was considered stable when (1) the proportion of highreward choices made in any of the final five sessions did not deviate from the mean of those sessions by more than $15 \%$ and (2) there was no visually apparent trend in choice over the final five sessions. If choice was not stable after 30 sessions, the phase was terminated when the second criterion alone was met. 
Probe sessions. Two LE rats consistently omitted all forced- and/or free-choices on the high-reward lever during the final trial block (FR 60). For these rats only, the third trial block (FR 30) was omitted during probe sessions, under the rationale that fewer pellets would have been consumed by the third block and this may increase responding in the FR 60 block. One rat completed two probe sessions, the other completed four.

Effort-Based Impulsive Choice Test II. Although time to food on both alternatives was always equal in Test I, the contingency did result in varying interreinforcer intervals across rats, since some rats were faster at completing the HR FRs than others. This issue was addressed in Test II. Rats completed the same assessments as described in Effort-Based Impulsive Choice Test I, except that average inter-reinforcer intervals were held constant across the HE and LE groups. To accomplish this, the postfood blackout was programmed to vary in duration so that new trials were initiated every $30,60,90$, or $120 \mathrm{~s}$ in the four trial blocks, respectively. Rats completed at least 5 sessions in this phase, and until the stability criteria were met (described above).

Return to Effort Training. Because no lasting group differences were detected in the first two impulsive choice assessments, rats were returned to their respective training (HE or LE) for thirty sessions before completing a final, modified effort-based impulsive choice assessment. HE and LE training sessions were the same as described previously, except trial durations were held constant at $60 \mathrm{~s}$ for all rats.

Effort-Based Impulsive Choice Test III. Rats completed the same assessment as described in Effort-Based Impulsive Choice Test II, except that reinforcer amounts were 2 pellets (LR) or 4 pellets (HR). In prior phases, all rats chose the larger, more effortful reward much more than expected based on the extant literature. Therefore, I adopted the 
2- vs. 4-pellet amounts more commonly arranged (e.g., Floresco et al., 2008). Rats

completed at least 10 sessions of this phase, and continued until they met the previously described stability criteria. If choice was not stable by session 25 , the phase was terminated once there was no visually apparent trend across the final 5 sessions.

\section{Data Analysis}

Distributions of several non-choice measures violated assumptions of normality (number of trials completed in training sessions, number of sessions to pass amount discrimination, number of returns to amount discrimination testing, and total number of omissions across analyzed sessions in Effort-Based Impulsive Choice Test I). Thus, Mann-Whitney $U$ tests were used to evaluate group differences in non-choice measures. Area under the curve (AUC; Myerson, Green, \& Warusawitharana, 2001) over the final five stable sessions served as the dependent measure of effort-based impulsive choice; larger AUC values reflect less impulsive choice. Beta regression was used to analyze group differences in each test of impulsive choice, and Pseudo $\mathrm{R}^{2}$ was used to evaluate variability accounted for by the independent variable. All analyses were conducted in in $R$ (R Core Team, 2013) using the "betareg" package (Ferrari \& Cribari-Neto, 2004). Nonparametric test statistics for nonchoice group comparisons were obtained using the "furniture" package (Barrett \& Brignone, 2017) in $R$. To evaluate the possibility of transient group differences, supplemental comparisons were conducted on choices made in the initial five sessions of Effort-Based Impulsive Choice Tests I and III (the two tests immediately following HE/LE training). ${ }^{2}$ Two LE rats consistently omitted all trials in

\footnotetext{
${ }^{2}$ When rats chose the high-reward alternative fewer than $80 \%$ of the time in the first trial block (FR1) data from that session were not included in the analysis (sessions were omitted for $2 \mathrm{HE}$ rats, and $8 \mathrm{LE}$ rats). In other words, the first five sessions wherein the high-reward was selected $\geq 80 \%$ of the time in the first trial block were analyzed.
} 
the third and/or fourth trial blocks of the effort-based impulsive choice assessments. To impute these missing data, I explored linear and nonlinear functions that could fit choices made in the first three trial blocks, and predict obtained choices in the fourth trial block, among rats with complete datasets. A linear function, with the FR1 intercept constrained to $100 \%$, provided the best predictions, deviating from the obtained data by an average of $6.6 \%$. This function was fit to the two rats with missing data to impute choice in the final trial block, which was needed to calculate AUC.

\section{Results}

HE and LE groups did not differ in days to acquire lever-pressing $(p=.47)$, or in the number of HE or LE trials completed during training $(p>.99)$ or retraining $(p>.4)$. HE and LE rats did not differ in number of sessions to pass amount discrimination ( $p=$ $.15)$, but did differ in number of returns to amount discrimination testing $(p=.04)$, with LE rats requiring more returns to amount discrimination. Additionally, LE rats had significantly more omissions across analyzed sessions in Effort-Based Impulsive Choice Test I $(p<.01)$.

Figure 2 shows AUC values from the initial five sessions of Effort-Based Impulsive Choice Tests I and III. HE training was a significant predictor of AUC in both Effort-Based Impulsive Choice Test I $\left(p<.0001\right.$; Pseudo $\left.\mathrm{R}^{2}=0.25\right)$ and Test III $(p=$ .001 ; Pseudo $\left.\mathrm{R}^{2}=0.186\right)$, both directly following HE/LE training.

Figure 3 shows the results of HE and LE training on AUC in the final five sessions of Effort-Based Impulsive Choice Tests I, II, and III. There was no lasting effect of effort training on AUC observed in the final five sessions of Effort-Based Impulsive Choice Test I $(p=.106)$ or Test II $(p=.203)$, but group remained a significant predictor 
of AUC in Test III $\left(p=.032\right.$; Pseudo $\left.\mathrm{R}^{2}=.12\right)$, with HE rats showing a greater preference for the high-effort, high reward choice.

\section{Discussion}

The current study was conducted to assess the effects of high-effort training on effort-based impulsive choice, with proper delay controls, at a range of effort requirements. Although an initial effect of effort training was observed in the first five sessions of Effort-Based Impulsive Choice Tests I and III, a lasting effect of HE training was observed in only one phase (Test III) and the magnitude of the effect was small. Overall, by the final five sessions in each test phase, a ceiling effect was observed and most rats preferred the high-effort high reward choice. This finding replicates prior studies that have evaluated short-term effects of effort training (Eisenberger \& Adornetto, 1986; Eisenberger et al., 1985; Eisenberger et al., 1989) and extends the literature by analyzing choice over a longer test phase. Below I outline three possible explanations for the overall ceiling effect observed, and 2 potential reasons for the lack of a lasting effect in Effort-Based Impulsive Choice Tests I and II.

One possible account for the overall ceiling effect in impulsive choice is that the effort requirements used in this study differed from previous studies of the effortdiscounting with equivalent delays task (Floresco et al., 2008; Ghod-Sharifi \& Floresco, 2010; Ghods-Sharifi, Onge, \& Floresco, 2009; Shafiei, Gray, Viau, \& Floresco, 2012). Floresco et al. (2008) used FR requirements of 2, 5, 10, and 20, while the current study employed a wider range of FR requirements $(1,15,30$, and 60$)$. Because time to food on the low-reward alternative was yoked to the time it took to complete the high-reward FR, larger FRs entailed longer delays to the small food reward. This may have increased 
preference for the larger, more-effortful reward, and thus, may have produced the ceiling effect.

A second potential contributing factor to the observed ceiling effect in impulsive choice relates to the difference in reward magnitudes initially arranged during the effortbased impulsive choice assessment. In Effort-Based Impulsive Choice Tests I and II, rats chose between 1 and 3 pellets; however, previous studies that reported more robust effort discounting arranged 2 vs. 4 pellets (e.g. Floresco et al., 2008). Weber's law (also called the Weber-Fechner law), explains that the perceived value or strength of a stimulus is proportional to its magnitude (Hecht, 1924; Namboodiri, Mihalas, \& Shuler, 2014). Accordingly, a 3-pellet reward is three times more valuable than a 1-pellet reward, but a 4-pellet reward has only twice the value of a 2-pellet reward. Thus, in Choice Tests I and II, the perceived difference in reward amount was larger than in past studies of effort discounting, and this may have increased preference for the larger, more-effortful alternative. In Choice Test III, the reward amounts were changed to 2 and 4 pellets. The results of Test III provide additional evidence that the initial reward magnitudes played a role in the observed ceiling effect, as Test III was the only phase in which a lasting training effect was observed.

A third potential explanation for the ceiling effect is that the long delays to food on the low-reward alternative could have resulted in indistinguishable assignment of credit (i.e., difficulty in response-reward contingency learning; Killeen, 2011; Niv, Joel, Meilijson, \& Ruppin, 2002; Sutton \& Barto, 1990). The contingency on the high-reward lever ensured that food always immediately followed a lever press. By contrast, on the low-reward lever, a signaled delay was imposed between press and reward. Response- 
reward relations are more difficult to learn when reinforcement is delayed (e.g., Black, Belluzzi, \& Stein, 1985; Perin, 1943) and this may have increased preference for the high-effort alternative, and contributed to the ceiling effect.

An issue related to the ceiling effect is why HE training did not produce a lasting reduction in effort-based impulsive choice, relative to LE training. One possible account is that the number of forced-choice trials that I arranged in the tests of impulsive choice is greater than past studies of effort discounting (e.g., Floresco et al., 2008). Forced-choice trials on the high-effort alternative may have rapidly produced a tolerance for high effort; i.e., low effort discounting. In every five sessions of effort-based impulsive choice assessments, rats completed up to 45 trials of FR requirements ranging from 15 to 60 , which might have had the effect of reducing effort-based impulsive choice. Evidence for this possibility is that a temporary effect of HE training was observed in Choice Tests I and III. The effect of training was smaller in the initial sessions of Choice Test III than in Choice Test I (i.e., Pseudo $\mathrm{R}^{2}=0.186$ and 0.25 , respectively) perhaps because of LE rats' prior "training" with forced-choice high-effort trials in Choice Tests I and II. This rapidacquisition of HE training hypothesis is consistent with the results of Eisenberger et al. (1989). In that experiment, group differences in effort discounting were observed after just 18 sessions of HE or LE training, with the former group completing only 20 trials at the terminal effort requirement. These group differences appeared to be fading after approximately 10 sessions, suggesting that LE rats were acquiring effort tolerance with continued testing. Testing was terminated at session 12, so it is unknown if the group difference would have maintained with additional sessions. 
A separate literature suggests that rewards which follow higher effort acquire a higher value than rewards obtained by less effort (e.g., Kacelnik \& Marsh, 2002; Klein, Bhatt, \& Zentall, 2005; Tsukamoto \& Kohara, 2017; Tsukamoto, Kohara, \& Takeuchi, 2017; Zentall \& Singer, 2007). This phenomenon is called the within-trial contrast effect (Zentall \& Singer, 2007). Applied to my experiment, the value of the high-effort reward would be enhanced by this contrast effect, whereas the value of the low-effort reward would be unchanged. Because I employed more forced-choices than Floresco et al. (2008), there were more opportunities to develop a within-trial contrast effect in my experiment than in Floresco's. If rats valued the high-reward more than the low-reward, in part, because of a within-trial contrast effect, then this may have contributed to the ceiling effect. The initial effect of LE rats selecting the low-reward more frequently than HE rats may have quickly diminished due to forced-choice exposure to high-effort contingencies, resulting in an increase in value assigned to the high-reward alternative.

Two limitations of the present study are noteworthy. First, there was no true control group. Half of the rats were assigned to HE, and half were assigned to LE. A control group that experienced no effort training may have clarified whether the surprising effort tolerance in the LE group was due to extensive training on an FR1, or to other factors (e.g., high-effort forced choices, long delays to low-reward). Second, all rats experienced the test phases in the same order. A larger effect may have maintained over time had Choice Test III ( 2 vs. 4 pellets) been conducted first. It is impossible to evaluate whether any of the observed findings were due, in part, to a sequence effect. Future research should aim to address these limitations by including a no-training control group and by counterbalancing test phases. Additional research in this area may also aim 
to determine the speed of high effort acquisition, identify interventions to harness that quick acquisition, and test for generalization and maintenance of effects. 


\section{References}

Alessi, S.M., \& Petry, N.M. (2003). Pathological gambling severity is associated with impulsivity in a delay discounting procedure. Behavioural Processes, 64, $345-354$.

Białaszek, W., Marcowski, P., \& Ostaszewski, P. (2017). Physical and cognitive effort discounting across different reward magnitudes: Tests of discounting models._PloS One,-12(7), e0182353. doi: 10.1371/journal.pone.0182353 Black, J., Belluzzi, J. D., \& Stein, L. (1985). Reinforcement delay of one second severely impairs acquisition of brain self-stimulation._-Brain research,_-359(1-2), 113119.

Boyagian, L. G., \& Nation, J. R. (1981). The effects of force training and reinforcement schedules on human performance.-The American Journal of Psychology, 94(4), 619- 632.

Brandon, T. H., Herzog, T. A., Juliano, L. M., Irvin, J. E., Lazev, A. B., \& Simmons, V. N. (2003). Pretreatment task persistence predicts smoking cessation outcome.Journal of Abnormal Psychology,-112(3), 448-456.

Cosgrove KP, Hunter R, Carroll ME (2002) Wheel-running attenuates intravenous cocaine self- administration in rats: sex differences. Pharm Biochem Behav 73:663671.

Dixon, M. R., \& Holton, B. (2009). Altering the magnitude of delay discounting by pathological gamblers. Journal of Applied Behavior Analysis, 42(2), 269275. 
Dixon, M. R., Rehfeldt, R. A., \& Randich, L. (2003). Enhancing tolerance to delayed reinforcers: The role of intervening activities._-Journal of Applied

Behavior Analysis,-36(2), 263-266.

Dombrovski AY, Siegle GJ, Szanto K, Clark L, Reynolds CF, \& Aizenstein H. (2012).

The temptation of suicide: Striatal gray matter, discounting of delayed rewards, and suicide attempts in late-life depression. Psychological Medicine, 42, 1203-15.

Duckworth, A. L., Peterson, C., Matthews, M. D., \& Kelly, D. R. (2007). Grit:

perseverance and passion for long-term goals.- Journal of Personality and Social Psychology,_- 92(6),1087-1101.

Duckworth, A., \& Gross, J. J. (2014). Self-control and grit: Related but separable determinants of success.-Current Directions in Psychological Science,-23(5), $319-325$.

Dweck, C. S. (2010). Even geniuses work hard._Educational Leadership,_68(1), 1620.

Eisenberger, R. (1992). Learned industriousness._Psychological Review,_99(2), 248267.

Eisenberger, R., \& Adornetto, M. (1986). Generalized self-control of delay and effort.Journal of Personality and Social Psychology,_51(5), 1020-1031.

Eisenberger, R., Carlson, J., Guile, M., \& Shapiro, N. (1979a). Transfer of effort across behaviors.-Learning and Motivation,-10(2), 178-197.

Eisenberger, R., Heerdt, W. A., Hamdi, M., Zimet, S., \& Bruckmeir, G. (1979b). Transfer of persistence across behaviors._-Journal of Experimental Psychology:

Human Learning $\quad$ and Memory, - 5(5), 522-530. 
Eisenberger, R., \& Leonard, J. M. (1980). Effects of conceptual task difficulty on generalized persistence. American Journal of Psychology, 93, 285-298.

Eisenberger, R., Leonard, J. M., Carlson, I, \& Park, D. C. (1979c). Transfer effects of contingent and noncontingent positive reinforcement: Mechanisms and generality. American Journal of Psychology, 92, 525-535.

Eisenberger, R., \& Masterson, F. A. (1983). Required high effort increases subsequent persistence and reduces cheating. Journal of Personality and Social Psychology, 44, $593-599$.

Eisenberger, R., Masterson, F. A., \& McDermitt, M. (1982). Effects of task variety on generalized effort._Journal of Educational Psychology,_74(4), 499-505.

Eisenberger, R., Mitchell, M., \& Masterson, F. A. (1985). Effort training increases generalized self-control._-Journal of Personality and Social Psychology,49(5), 1294-1301.

Eisenberger, R., Terborg, R., \& Carlson, J. (1979). Transfer of persistence across reinforced $\quad$ behaviors. Animal Learning and Behavior, 40, 493-498.

Eisenberger, R., Weier, F., Masterson, F. A., \& Theis, L. Y. (1989). Fixed-ratio schedules increase generalized self-control: Preference for large rewards despite high

effort or punishment._-Journal of Experimental Psychology: Animal

Behavior Processes,-15(4), 383-392.

Evenden, J. L., \& Ryan, C. N. (1996). The pharmacology of impulsive behaviour in rats: The effects of drugs on response choice with varying delays of reinforcement.Psychopharmacology,-128(2), 161-170. 
Eubig, P. A., Noe, T. E., Floresco, S. B., Sable, J. J., \& Schantz, S. L. (2014). Sex

differences in

delay-discounting response to amphetamine in adult Long-Evans rats performing a task.-Pharmacology Biochemistry and Behavior,-118,

1-9.

Flora, S. R., \& Pavlik, W. B. (1990). Conventional and reversed partial reinforcement

effects in human operant responding._-Bulletin of the Psychonomic Society,_-28(5), 429-432.

Floresco, S. B., Maric, T. L., \& Ghods-Sharifi, S. (2008). Dopaminergic and glutamatergic regulation of effort-and delay-based decision making.Neuropsychopharmacology,-_ 33(8), 1966-1979.

Fox, J., \& Weisberg, S. (2002). An \{R $\}$ Companion to Applied Regression (2nd Edition). Thousand Oaks, CA: Sage.

Ghods-Sharifi, S., \& Floresco, S. B. (2010). Differential effects on effort discounting induced by inactivations of the nucleus accumbens core or shell.-Behavioral Neuroscience,- $\quad$ 124(2), 179-191.

Ghods-Sharifi, S., Onge, J. R. S., \& Floresco, S. B. (2009). Fundamental contribution by the basolateral amygdala to different forms of decision making.-Journal of Neuroscience,-29(16), 5251-5259.

Gray, J.C., \& MacKillop, J. (2015). Impulsive delayed reward discounting as a genetically -influenced target for drug abuse prevention: A critical evaluation. Frontiers in Psychology, 6, 1104. doi: 10.3389/fpsyg.2015.01104 Hecht, S. (1924). The visual discrimination of intensity and the Weber-Fechner law.The Journal of general physiology,-7(2), 235-267. 
Hodos, W. (1961). Progressive ratio as a measure of reward strength._-Science,134(3483), 943-944.

Kacelnik, A., \& Marsh, B. (2002). Cost can increase preference in starlings. Animal

Behaviour, $\quad 63,245-250$. http://dx.doi.org/10.1006/anbe .2001.1900

Killeen, P.R. (2011). Models of trace decay, eligibility for reinforcement, and delay of reinforcement gradients, from exponential to hyperboloid. Behavioural Processes, $87, \quad 57-63$.

Klein, E. D., Bhatt, R. S., \& Zentall, T. R. (2005). Contrast and the justification of effort. Psychonomic Bulletin \& Review, 12, 335-339.

http://dx.doi.org/10.3758/BF03196381

Koffarnus MN, Jarmolowicz DP, Mueller ET, \& Bickel WK. (2013). Changing delay discounting in the light of the competing neurobehavioral decision systems theory: A review. Journal of the Experimental Analysis of Behavior, 99, 32-57.

Kowal, B. P., \& Faulkner, J. L. (2016). Delay discounting of hypothetical monetary rewards with decoys. Behavioural Processes, 122, 26-35.

Larson EB, Carroll ME (2005) Wheel running as a predictor of cocaine selfadministration and reinstatement in female rats. Pharm Biochem Behav 82:590 600.

Logue, A. W., \& Mazur, J. (1981). Maintenance of self-control acquired through a fading procedure: follow-up on Mazur and Logue (1978). Behavior Analysis Letters, 1, 131-137.

Logue, A. W., Smith, M. E., \& Rachlin, H. (1985). Sensitivity of pigeons to prereinforcer and postreinforcer delay. Animal Learning and Behavior, 13, 181-186. 
Malesza, M., \& Ostaszewski, P. (2013). Relations between Cloninger's dimensions of temperament and steepness of delay and effort discounting of monetary rewards.- Psychological Reports,-112(3), 694-705.

Mazur, J.E. (1988). Choice between small certain and large uncertain reinforcers. Animal Learning and Behavior, 16, 199-205.

Mazur, J. E. (2012). Effects of pre-trial response requirements on self-control choices by rats and pigeons. Journal of the Experimental Analysis of Behavior, 97(2), 215230.

McClure, SM, Laibson, DI, Loewenstein, G, Cohen, JD (2004). Separate neural systems value immediate and delayed monetary rewards._-Science 306(5695),_503507.

Mitchell, S. H. (1999). Measures of impulsivity in cigarette smokers and nonsmokers. Psychopharmacology, 146, 455-464.

Mitchell, S. H. (2004). Effects of short-term nicotine deprivation on decision-making: delay, uncertainty and effort discounting.-Nicotine \& Tobacco Research,_6(5), 819828.

Myerson, J., Green, L., \& Warusawitharana, M. (2001). Area under the curve as a measure of discounting.- Journal of the Experimental Analysis of Behavior,$76(2), 235-243$

Nation, J. R., Cooney, J. B., \& Gartrell, K. E. (1979). Durability and generalizability of persistence training.-Journal of Abnormal Psychology,_-88(2), 121-136.

Namboodiri, V. M. K., Mihalas, S., \& Hussain Shuler, M. G. (2014). A temporal basis for Weber's law in value perception._-Frontiers in Integrative Neuroscience,_-8, 79. 
Niv, Y., Joel, D., Meilijson, I., \& Ruppin, E. (2002). Evolution of reinforcement learning in uncertain environments: A simple explanation for complex foraging behavior. Adaptive Behavior, 10, 5-24.

Odum, A. L. (2011). Delay discounting: Tait variable?_Behavioural Processes,_87(1), $1-9$.

Odum, A. L. (2011). Delay discounting: I'm a $k$, you're a $k$.- Journal of the Experimental Analysis of Behavior,-96(3), 427-439.

Orsini, C. A., \& Setlow, B. (2017). Sex differences in animal models of decision making._Journal of Neuroscience Research,_95(1-2), 260-269.

Paglieri, F. (2013). The costs of delay: Waiting versus postponing in intertemporal choice.- Journal of the Experimental Analysis of Behavior,_-99(3), 362-377.

Phung, Q. H., Snider, S. E., Tegge, A. N., \& Bickel, W. K. (2019). Willing to work but not to wait: Individuals with greater alcohol use disorder show increased delay discounting across commodities and less effort discounting for alcohol._-Alcoholism: Clinical and Experimental Research,-43(5), 927-936.

Perin, C. T. (1943). A quantitative investigation of the delay-of-reinforcement gradient._ - Journal of Experimental Psychology,_-32(1), 37.

Pinkston, J. W., \& Libman, B. M. (2017). Aversive functions of response effort: Fact or artifact?-Journal of the Experimental Analysis of Behavior,-108(1), 73-96.

Pittenger, D. J., \& Pavlik, W. B. (1988). Analysis of the partial reinforcement extinction effect in humans using absolute and relative comparisons of schedules. - The American Journal of Psychology, 101(1), 1-14. 
Quinn, E. P., Brandon, T. H., \& Copeland, A. L. (1996). Is task persistence related to smoking and substance abuse? The application of learned industriousness theory to addictive behaviors._Experimental and Clinical Psychopharmacology,$4(2), 186-190$.

Renda, C. R., \& Madden, G. J. (2016). Impulsive choice and pre-exposure to delays: III. Four -month test-retest outcomes in male Wistar rats.-Behavioural Processes,-126, $108-112$.

Renda, C., Rung, J. M., Hinnenkamp, J. E., Lenzini, S. N., \& Madden, G. J. (2018). Impulsive choice and pre-exposure to delays: iv. effects of delay-and immediacyexposure training relative to maturational changes in impulsivity.-Journal of the Experimental Analysis of Behavior,-109(3), 587-599.

Rung, J. M., Buhusi, C. V., \& Madden, G. J. (2018). Reducing impulsive choice: V. The role of timing in delay-exposure training._Behavioural Processes. doi: 10.1016/j.beproc.2018.04.018

Rung, J. M., \& Madden, G. J. (2018). Experimental reductions of delay discounting and impulsive choice: A comprehensive review and meta-analysis. Journal of Experimental Psychology: General.

Seaman, K. L., Brooks, N., Karrer, T. M., Castrellon, J. J., Perkins, S. F., Dang, L. C., ... \& Samanez-Larkin, G. R. (2018). Subjective value representations during effort, probability and time discounting across adulthood.-Social Cognitive and Affective Neuroscience,-13(5), 449-459. 
Shafiei, N., Gray, M., Viau, V., \& Floresco, S. B. (2012). Acute stress induces selective alterations in cost/benefit decision-making.-Neuropsychopharmacology,37(10), 2194- 2209.

Smith, A. P., Marshall, A. T., \& Kirkpatrick, K. (2015). Mechanisms of impulsive choice: II. Time-based interventions to improve self-control. Behavioural Processes, 112, 29-42.

Smith, T., Panfil, K., Bailey, C., \& Kirkpatrick, K. (2019). Cognitive and behavioral training interventions to promote self-control._-Journal of Experimental Psychology: Animal Learning and Cognition, 45(3), 259-279.

Stein, J. S., Johnson, P. S., Smits, R. R., Renda, R., Liston, K. J., Shahan, T. S., \& Madden, G. J. (2013). Early and prolonged exposure to reward delay: Effects on impulsive choice and alcohol self-administration in male rats. Experimental and Clinical Psychopharmacology, $\quad 21,172-180$

Stein, J.S., \& Madden, G.J., \& (2013). Delay discounting and drug abuse: Empirical, conceptual, $\quad$ and methodological considerations. In H. de Wit \& J. MacKillop (Eds.), The Wiley- Blackwell Handbook of Addiction Psychopharmacology. Boston, MA: Wiley-Blackwell (pp. 165-208).

Stein, J. R., Renda, C. R., Hinnenkamp, J., \& Madden, G. J. (2015). Impulsive choice, alcohol consumption, and pre-exposure to delayed rewards II: Potential mechanisms. Journal of $\quad$ the Experimental Analysis of Behavior, 103, 33-49. Sugiwaka, H., \& Okouchi, H. (2004). Reformative self-control and discounting of reward value $\quad$ by delay or effort._Japanese Psychological Research,_46(1), 1-9. Sutton, R. S., \& Barto, A. G. (1990). Time-derivative models of pavlovian reinforcement. 
Tsukamoto, M., \& Kohara, K. (2017). Using the implicit association test and choice to measure the within-trial contrast effect in human adults. The Psychological Record, 67, 507-518. http://dx.doi.org/10.1007/ s40732-017-0253-0

Tsukamoto, M., Kohara, K., \& Takeuchi, K. (2017). Effects of effort and difficulty on human preference for a stimulus: Investigation of the within-trial contrast. Learning \& Behavior, $\quad 45,135-146$. http://dx.doi .org/10.3758/s13420-016-0248-8 van der Wal, A. J., Schade, H. M., Krabbendam, L., van Vugt, M., Wal, A. J. van der, Schade, H. M., ... Vugt, M. van. (2013). Do natural landscapes reduce future discounting in humans? Proceedings. Biological Sciences / The Royal Society, 280(1773), 20132295- 20132295. doi: 10.1098/rspb.2013.2295

Weiner, B. (1985). An attributional theory of achievement motivation and emotion.-Psychological Review,-92(4), 548-573.

Weller, R. E., Cook, E. W., Avsar, K. B., \& Cox, J. E. (2008). Obese women show greater delay discounting than healthy-weight women. Appetite, 51, 563-569. Wenrich, W. W., Eckman, G. E., Moore, M. J., \& Houston, D. F. (1967). A transresponse effect of partial reinforcement.—Psychonomic Science,_9(5), 247-248. Wills, T. A., Sandy, J. M., \& Yaeger, A. M. (2001). Time perspective and early-onset substance use: A model based on stress-coping theory.-Psychology of Addictive Behaviors,-15(2), 118-125.

Winstanley, C. A., Theobald, D. E., Cardinal, R. N., \& Robbins, T. W. (2004).

Contrasting roles of basolateral amygdala and orbitofrontal cortex in impulsive choice.-Journal of Neuroscience,-24(20), 4718-4722. 
Wu, C. Y., \& He, G. B. (2012). The effects of time perspective and salience of possible monetary losses on intertemporal choice. Social Behavior and Personality, $40(10), 1645-1654$.

Young, M. E. (2018). Discounting: A practical guide to multilevel analysis of choice data.- Journal of the Experimental Analysis of Behavior,-109(2), 293-

312.

Zentall, T. R., \& Singer, R. A. (2007). Within-trial contrast: Pigeons prefer conditioned reinforcers that follow a relatively more rather than a less aversive event. Journal of the Experimental Analysis of Behavior, 88, 131-149.

http://dx.doi.org/10.1901/jeab.2007.27- 06

Zlebnik NE, Carroll ME (2015a) Effects of the combination of wheel running and atomoxetine on cue-and cocaine-primed reinstatement in rats selected for high or low impulsivity. Psychopharmacol 232:1049-59.

Zlebnik NE, Carroll ME (2015b) Prevention of incubation of cocaine seeking by physical exercise in female rats. Psychopharmacology (Berl)2015 Jul 11. PMID:

26159456. Psychopharmacology 232(19):3507-13. 
Figure 1. A visual representation of the effort-based impulsive choice task, based on Floresco et al. (2008).

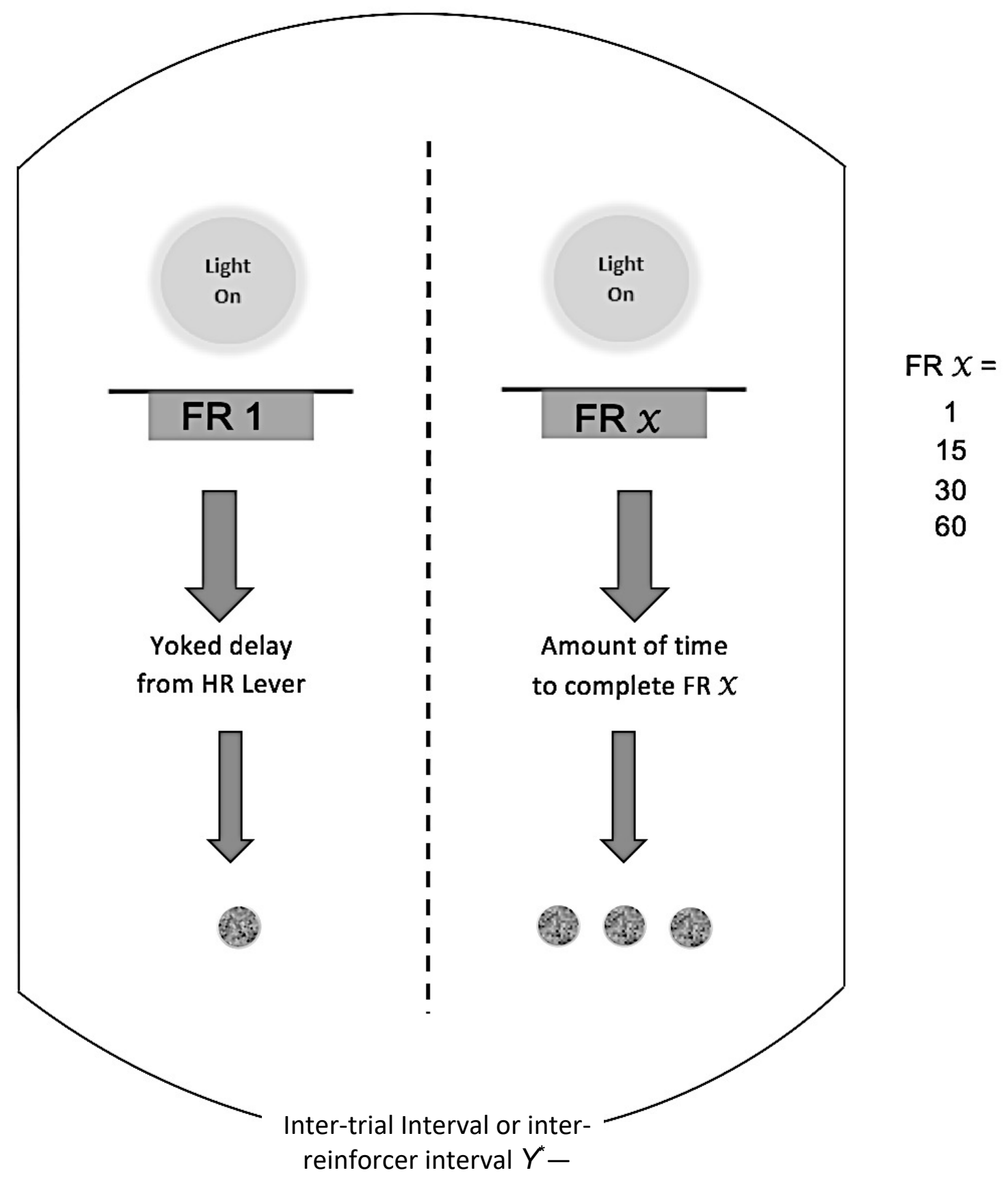

*Trial durations varied across effort-based impulsive choice conditions, see methods for details. 
Figure 2. Average area under the choice curve (AUC) for HE and LE rats in the initial five sessions of Effort-Based Impulsive Choice Test I (top panel) and Test III (bottom panel), both of which directly followed HE/LE training. Error bars indicate the standard deviation.

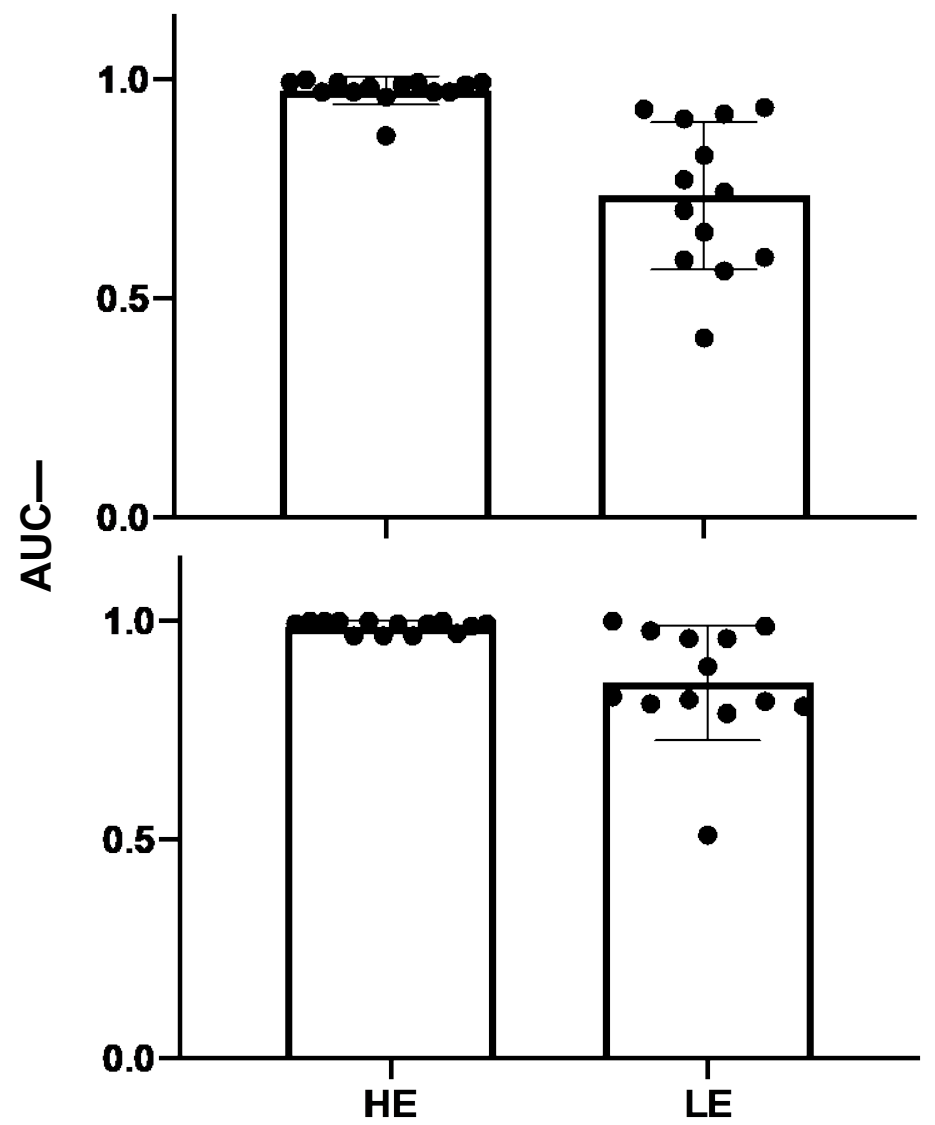


Figure 3. Average AUC for HE and LE rats in the stable sessions of Effort-Based Impulsive Choice Test I (top panel), Test II (middle panel), and Test III (bottom panel). Error bars indicate the standard deviation.

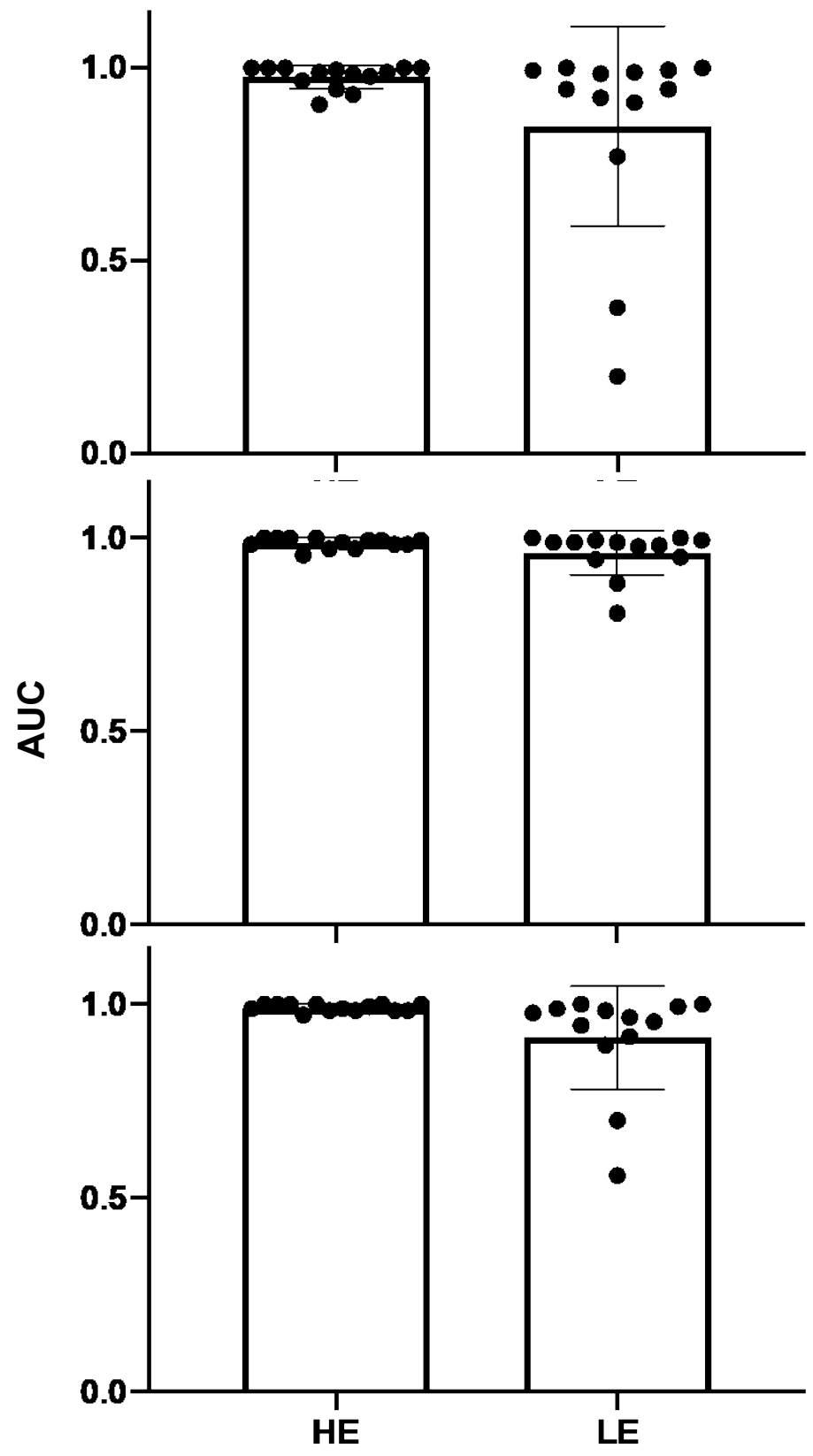




\section{Appendix A}

Yoked delays (s) for the low-reward alternative in trial blocks 2, 3, and 4 for high effort (HE) and low effort (LE) rats across the final five sessions of Effort-Based Impulsive Choice Test I. Dashes indicate missing data, due to rats failing to complete forced choices in a given trial block.

\begin{tabular}{|c|c|c|c|c|c|}
\hline \multirow[b]{2}{*}{ Rat \# } & \\
\hline & 1 & 2 & 3 & 4 & 5 \\
\hline 1 & 3.42 & 2.84 & 3.32 & 3.47 & 20.13 \\
\hline 2 & 4.16 & 5.04 & 5.72 & 4.71 & 4.77 \\
\hline 3 & 4.44 & 4.93 & 4.21 & 5.26 & 5.05 \\
\hline 4 & 4.46 & 4.38 & 3.51 & 4.19 & 4.16 \\
\hline 5 & 7.53 & 8.59 & 8.85 & 8.04 & 8.91 \\
\hline 6 & 6.38 & 5.54 & 5.54 & 5.83 & 6 \\
\hline 7 & 4.73 & 4.95 & 4.77 & 4.14 & 4.73 \\
\hline 8 & 5.15 & 3.97 & 4.34 & 4.07 & 4.45 \\
\hline 9 & 5.4 & 6.66 & 5.37 & 6.45 & 6.66 \\
\hline 10 & 3.55 & 3.42 & 3.97 & 3.27 & 4.62 \\
\hline 11 & 4.61 & 4.79 & 3.2 & 14.63 & 4.13 \\
\hline 12 & 4.85 & 5.71 & 6.09 & 5.99 & 6.78 \\
\hline 13 & 4.28 & 4.03 & 3.39 & 4.04 & 4.42 \\
\hline \multirow[t]{2}{*}{14} & 4.03 & 4.33 & 3.9 & 4.07 & 4.05 \\
\hline & \multicolumn{5}{|c|}{ HE Final 5 Sessions Trial block 3 (FR 30): } \\
\hline Rat \# & 1 & 2 & 3 & 4 & 5 \\
\hline 1 & 7.24 & 6.95 & 7.87 & 7.47 & 18.46 \\
\hline 2 & 9.58 & 9.4 & 9.13 & 9.94 & 10.81 \\
\hline 3 & 9.84 & 8.68 & 7.41 & 7.84 & 7.99 \\
\hline 4 & 6.71 & 6.29 & 6.06 & 5.82 & 6.08 \\
\hline 5 & 17.47 & 16.18 & 15.38 & 17.11 & 16.05 \\
\hline 6 & 12.14 & 9.94 & 10.16 & 11.4 & 14.18 \\
\hline 7 & 8.65 & 9.56 & 9.91 & 11.01 & 10.25 \\
\hline 8 & 9.73 & 10.86 & 10.23 & 9.17 & 9.5 \\
\hline 9 & 16.3 & 12.95 & 13.47 & 15.32 & 10.87 \\
\hline 10 & 8.53 & 7.45 & 8.5 & 8.5 & 9.95 \\
\hline 11 & 10.47 & 7.25 & 4.34 & 16.23 & 9.49 \\
\hline 12 & 10.75 & 11.34 & 10.91 & 10.56 & 12.06 \\
\hline
\end{tabular}




$\begin{array}{llllll}13 & 6.45 & 6.26 & 5.98 & 7.48 & 6.95 \\ 14 & 7.82 & 5.94 & 6.65 & 8.56 & 7.19\end{array}$

HE Final 5 Sessions Trial block 4 (FR 60):

$\begin{array}{cccccc}\text { Rat \# } & \mathbf{1} & \mathbf{2} & \mathbf{3} & \mathbf{4} & \mathbf{5} \\ \mathbf{1} & 15.91 & 16.48 & 16.84 & 15.53 & 34.65 \\ \mathbf{2} & 19.49 & 19.09 & 20.97 & 20.43 & 20.89 \\ \mathbf{3} & 19.19 & 18.97 & 18.73 & 15.69 & 17.66 \\ \mathbf{4} & 15.16 & 10.09 & 11.92 & 12.11 & 11.99 \\ \mathbf{5} & 36.77 & 29.34 & 35.63 & 38.32 & 40.04 \\ \mathbf{6} & 23.05 & 18.14 & 23.93 & 22.99 & 23.71 \\ \mathbf{7} & 19.68 & 21.33 & 21.2 & 22.31 & 19.62 \\ \mathbf{8} & 20.92 & 20.69 & 20.77 & 19.1 & 19.64 \\ \mathbf{9} & 31.23 & 25.08 & 25.59 & 27.31 & 23.74 \\ \mathbf{1 0} & 21.99 & 16.49 & 16.29 & 16.21 & 24.31 \\ \mathbf{1 1} & 27.98 & 13.22 & 13.1 & 44.93 & 19.66 \\ \mathbf{1 2} & 22.06 & 28.01 & 21.44 & 22.28 & 26.58 \\ \mathbf{1 3} & 13.5 & 14.13 & 14.56 & 16.06 & 14.53 \\ \mathbf{1 4} & 16.22 & 15.83 & 13.57 & 14.22 & 13.68\end{array}$

LE Final 5 Sessions Trial block 2 (FR 15):

$\begin{array}{cccccc}\text { Rat \# } & \mathbf{1} & \mathbf{2} & \mathbf{3} & \mathbf{4} & \mathbf{5} \\ \mathbf{1} & 11.51 & 11.75 & 11.99 & 13.35 & 10.9 \\ \mathbf{2} & 7.36 & 8.57 & 6.69 & 9.3 & 7.76 \\ \mathbf{3} & 8.3 & 9.23 & 7.15 & 8.83 & 8.04 \\ \mathbf{4} & 5.94 & 5 & 5.89 & 5.81 & 6.21 \\ \mathbf{5} & 6.47 & 5.87 & 5.99 & 6.78 & 6.25 \\ \mathbf{6} & 8.22 & 7.23 & 8.11 & 8.81 & 8.39 \\ \mathbf{7} & 4.66 & 5.91 & 4.68 & 4.85 & 5 \\ \mathbf{8} & 8.58 & 10.25 & 9.19 & 8.76 & 8.39 \\ \mathbf{9} & 9.1 & 9.37 & 7.04 & 7.96 & 7.78 \\ \mathbf{1 0} & 7.85 & 7.84 & 8.51 & 8.19 & 8.05 \\ \mathbf{1 1} & 2.73 & 3.85 & 2.9 & 2.6 & 3.18 \\ \mathbf{1 2} & 7.51 & 9.78 & 8.03 & 8.43 & 7.61 \\ \mathbf{1 3} & 5.65 & 4.89 & 4.89 & 4.08 & 4.2\end{array}$

LE Final 5 Sessions Trial block 3 (FR 30):

Rat \#

1

2

3

4 


$\begin{array}{cccccc}\mathbf{1} & 29.13 & 35.72 & 36.58 & 36.61 & 41.48 \\ \mathbf{2} & 13.11 & 13.28 & 18.9 & 14.93 & 16.71 \\ \mathbf{3} & 18.8 & 20.1 & 22.55 & 20.87 & 20.33 \\ \mathbf{4} & 8.42 & 8.77 & 9.42 & 9.51 & 10.38 \\ \mathbf{5} & 13.09 & 12.37 & 10.83 & 11.62 & 10.99 \\ \mathbf{6} & 19.8 & 14.29 & 15.41 & 13.62 & 13.77 \\ \mathbf{7} & 10.31 & 8.74 & 9.88 & 10.19 & 10.66 \\ \mathbf{8} & 19.63 & 21.88 & 21.88 & - & 19.6 \\ \mathbf{9} & 16.31 & 20.08 & 13.48 & 12.64 & 15.12 \\ \mathbf{1 0} & 17.31 & 17.3 & 13.96 & 13.86 & 17.12 \\ \mathbf{1 1} & 4.83 & 6 & 5.86 & 6.43 & 5.87 \\ \mathbf{1 2} & 16.45 & 17.88 & 16.86 & 21 & 19.64 \\ \mathbf{1 3} & 13.72 & 12.11 & 10.78 & 11.14 & 9.59\end{array}$

LE Final 5 Sessions Trial block 4 (FR 60):

$\begin{array}{cccccc}\text { Rat \# } & \mathbf{1} & \mathbf{2} & \mathbf{3} & \mathbf{4} & \mathbf{5} \\ \mathbf{1} & \mathbf{-} & \mathbf{-} & \mathbf{-} & - & - \\ \mathbf{2} & 25.46 & 30.2 & 30.51 & 34.16 & 31.32 \\ \mathbf{3} & 43.35 & 45.46 & 54.44 & 52.43 & 40.75 \\ \mathbf{4} & 17.06 & 19.13 & 29.03 & 23.1 & 18.24 \\ \mathbf{5} & 24.03 & 29.35 & 22.95 & 21.61 & 24.4 \\ \mathbf{6} & 58.89 & 61.71 & 44.21 & 30.18 & 35.06 \\ \mathbf{7} & 22.68 & 23.61 & 22.34 & 24.15 & 21.63 \\ \mathbf{8} & - & - & - & - & - \\ \mathbf{9} & 40.75 & 32.9 & 33.84 & 28.99 & 26.26 \\ \mathbf{1 0} & 33.41 & 33.39 & 29.87 & 39.93 & 31.63 \\ \mathbf{1 1} & 13.64 & 14.38 & 14.34 & 18.35 & 15.05 \\ \mathbf{1 2} & 44.46 & 37.43 & - & 39.87 & 37.35 \\ \mathbf{1 3} & 27.36 & 35.58 & 27.01 & 62.94 & 21.86\end{array}$

